\title{
Phase diagram of trapped degenerate Fermi gases including effective s- and p-wave interactions
}

\author{
R. Roth and H. Feldmeier \\ Gesellschaft für Schwerionenforschung (GSI) \\ Planckstr. 1, 64291 Darmstadt, Germany \\ E-mail: r.roth@gsi.de, h.feldmeier@gsi.de
}

\begin{abstract}
The influence of s- and p-wave interactions on trapped degenerate one and two-component Fermi gases is investigated. The energy functional of a multicomponent Fermi gas is derived within the Thomas-Fermi approximation including the s- and p-wave terms of an effective contact interaction. On this basis the stability of the dilute gas against mean-field induced collapse due to attractive interactions is investigated and explicit stability conditions in terms of Fermi momentum and s- and p-wave scattering lengths are derived. Furthermore the spatial separation in a two-component Fermi gas is discussed, explicit conditions for the onset of component demixing are given, and the density distributions in the separated phase are calculated. The findings are summarized in a zerotemperature phase diagram for the degenerate two-component Fermi gas. It is shown that the p-wave interaction has significant influence on the phase diagram of the degenerate Fermi gas and causes new phenomena like absolute stabilization against collapse and component separation. It may therefore be useful in the context of the envisioned BCS transition in trapped atomic Fermi gases.
\end{abstract}

PACS numbers: 03.75.Fi, 34.20.Cf, 32.80.Pj

\section{Introduction}

Looking back at the outstanding experimental achievements in the field of trapped ultracold gases composed of bosonic atoms of the last 6 years one wonders what exciting new developments one has to envision in the near future [1]. One branch of these developments will involve dilute and degenerate gases of fermionic atoms. Already today several groups [2, 3, 任 were able to cool samples of fermionic atoms, ${ }^{6} \mathrm{Li}$ or ${ }^{40} \mathrm{~K}$, to temperatures corresponding to a fraction of the Fermi energy. These experiments use either a single fermionic isotope $\left({ }^{40} \mathrm{~K}\right)$ in two different hyperfine states [2] or a mixture of a fermionic and a bosonic species $\left({ }^{6} \mathrm{Li} /{ }^{7} \mathrm{Li}\right)$ [3, 4]. In this way s-wave scatterings between atoms of either the two fermionic components or between bosons and fermions allow efficient evaporative cooling of the gas. Remember that in a gas of identical fermions s-wave interactions are suppressed by the Pauli principle.

The experiments on dilute degenerate Fermi gases open a new and unique field to study fundamental quantum phenomena. Many of the questions discussed for the Bose-Einstein condensed Bose gas also apply to degenerate Fermi gases. Examples are the mean-field induced collapse of the dilute gas due to attractive interactions [5] or the variety of collective excitations [6]. One of the most challenging experimental goals is the realization of a BCS-transition from the normal to a superfluid phase 
[7. 8]. However, a detailed understanding of the "normal" degenerate Fermi gas is a prerequisite to achieve that.

In this paper we investigate the influence of interactions on single-component and two-component Fermi gases in the normal phase with special emphasis on the role of the p-wave interaction. Therefore in section 2 we construct an effective s- and p-wave contact interaction and use it to derive the energy density of a multicomponent Fermi gas in Thomas-Fermi approximation. On this basis we investigate the structure of one- and two-component Fermi gases at zero temperature. A first question concerns is the stability of the dilute gas against collapse induced by attractive interactions. In section 3 we derive stability conditions for the one- and two-component gas and discuss the influence of the p-wave interaction. A second question deals with the spatial separation of the different components in a two-component system. Conditions for the onset of component demixing are derived in section 1 . We also discuss the structure of the density distributions in the separated phase. Finally, in section 5 we summarize our findings by means of a zero-temperature phase diagram for the two-component Fermi gas.

\section{Energy functional of a multicomponent Fermi gas}

\subsection{Mean-field approximation and effective contact interaction}

In general the description of an interacting quantum mechanical many-body system relies on approximations to render the problem solvable. A general procedure to obtain different approximation schemes is to restrict the many-body states the system can assume to a certain subspace - called model space - of the full Hilbert space. A prominent example is the mean-field approximation where the many-body state is given by a symmetrized or antisymmetrized product of single-particle states. Within this mean-field model space the many-body problem can be solved; in case of fermions the solution is obtained by the well known Hartree-Fock equations.

However, this restriction to a model space of sufficiently simple structure encounters a serious drawback. Many realistic interactions between the constituents of the many-body system exhibit a strongly repulsive short-range core in addition to the attractive part. Typical examples are van der Waals interactions between neutral atoms, e.g. the Lennard-Jones potential with an attractive part proportional to $r^{-6}$ and a strong repulsion proportional to $r^{-12}$. The repulsive core prevents any of the particles to approach each other closer than the core radius, in other words, the twobody density distribution is depleted or even zero for particle distances smaller than the core radius. These short-range correlations cannot be described within a simple model space like the one of the mean-field approximation [9, 10].

To resolve this discrepancy we transform the realistic potential into an effective interaction suited for the particular model space under consideration. In the case of cold and dilute atomic gases the properties of the physical system simplify the construction of the effective interaction significantly. This is our starting point: (i) We want to describe the properties of a degenerate Fermi gas in the mean-field approximation, i.e. the many-body state is an antisymmetrized product state. (ii) The system is cold and dilute, i.e. mean particle distance and wavelength of relative motion are much larger than the range of the two-body potential. (iii) The system is in a non self-bound metastable many-body state, the two-body bound states of the potential are not populated. 
Due to (ii) and (iii) the atoms do not probe the detailed radial dependence of the interaction, therefore we can use a simple zero-range effective potential. To describe interactions also between particles with relative angular momenta $l>0$ the contact interaction has to be nonlocal. The mapping of the exact onto the effective interaction is done by means of the two-body energy spectrum: We require that the exact twobody eigenvalues $E_{n l}^{\text {exact }}$ for positive energies (scattering states) is reproduced by the expectation values of the effective interaction calculated with kinetic energy eigenstates $\left|n l m_{l}\right\rangle$

$$
\left\langle n l m_{l}\left|\frac{1}{2 m_{\mathrm{red}}} \overrightarrow{\boldsymbol{q}}^{2}+\boldsymbol{v}^{\mathrm{ECI}}\right| n l m_{l}\right\rangle \stackrel{!}{=} E_{n l}^{\text {exact }}
$$

where $\overrightarrow{\boldsymbol{q}}=\frac{1}{2}\left(\overrightarrow{\boldsymbol{p}}_{1}-\overrightarrow{\boldsymbol{p}}_{2}\right)$ is the relative momentum of the two particles and $m_{\text {red }}$ their reduced mass. In the following we will consider only particles with equal masses, i.e. $m_{\text {red }}=m / 2 . l$ and $m_{l}$ are the quantum numbers of relative angular momentum, and $n$ is a radial quantum number [11]. Guided by (11) we can construct the operator of the effective contact interaction (ECI) explicitly. Up to the quadratic order in the relative momentum $\vec{q}$, which includes a p-wave contribution, the operator of the ECI reads [1]

$\boldsymbol{v}^{\mathrm{ECI}}=\frac{4 \pi a_{0}}{m} \delta^{(3)}(\overrightarrow{\boldsymbol{r}})+\frac{6 \pi b_{0}}{m}\left[\delta^{(3)}(\overrightarrow{\boldsymbol{r}})(\hat{\boldsymbol{r}} \cdot \overrightarrow{\boldsymbol{q}})^{2}+\right.$ h.a. $]+\frac{12 \pi a_{1}^{3}}{m}(\overrightarrow{\boldsymbol{q}} \cdot \hat{\boldsymbol{r}}) \delta^{(3)}(\overrightarrow{\boldsymbol{r}})(\hat{\boldsymbol{r}} \cdot \overrightarrow{\boldsymbol{q}})$,

where $\hat{\boldsymbol{r}}=\boldsymbol{\boldsymbol { r }} /|\overrightarrow{\boldsymbol{r}}|$ and $\hbar=1$. The properties of the exact two-body interaction enter through the s-wave scattering length $a_{0}$, the s-wave effective volume $b_{0}$, and the pwave scattering length $a_{1}$. They are defined by the first terms of the low-momentum expansion of the scattering phase shifts $\eta_{l}(q)$ of the exact potential

$$
\frac{\tan \eta_{0}(q)}{q} \approx-a_{0}-b_{0} q^{2}, \quad \frac{\tan \eta_{1}(q)}{q^{3}} \approx-\frac{1}{3} a_{1}^{3} .
$$

The first term of the effective contact interaction (2) is identical to the widely used s-wave pseudopotential [12, 13. The two additional nonlocal terms are the natural extension to describe effective range effects and more important the effects of p-wave interactions.

One should be aware that the effective interaction (2) is designed for the application in a mean-field model space. It does not make sense to use this interaction in a larger model space or even for the solution of the Schrödinger equation [11]. When using erroneously this contact interaction to solve the exact scattering problem one does not get back the correct phase shifts.

\subsection{Energy density of a multicomponent Fermi gas}

We construct the energy density of the inhomogeneous Fermi gas in an external trapping potential within the Thomas-Fermi approximation, which was shown 14] to be an excellent approximation for the particle numbers considered here. The basic assumption of the Thomas-Fermi approximation is that the energy density of the inhomogeneous system is given locally by the energy density of the corresponding homogeneous system.

The calculation of the energy density of the homogeneous multicomponent Fermi gas in mean-field approximation is straightforward. The translational invariant part 
of the Hamiltonian of the trapped gas, consists of the kinetic energy operator and the effective contact interaction (2)

$$
\boldsymbol{H}_{\mathrm{hom}}=\frac{1}{2 m} \sum_{i}^{N} \overrightarrow{\boldsymbol{p}}_{i}^{2}+\sum_{i, j>i}^{N} \boldsymbol{v}_{i j}^{\mathrm{ECI}} .
$$

The energy density in mean-field approximation is defined by the expectation value of this Hamiltonian calculated with a Slater determinant $|\Psi\rangle$

$$
\mathcal{E}_{\text {hom }}=\frac{1}{V}\left\langle\Psi\left|\boldsymbol{H}_{\mathrm{hom}}\right| \Psi\right\rangle \text {. }
$$

The single-particles states $|i\rangle$ are eigenstates of the momentum operator with momenta $\vec{k}_{i}$ up to the Fermi momentum $\kappa$. To account for the different $m_{F}$ substates that can be trapped simultaneously [2] we characterize the single-particle states by an additional component quantum number $\xi=1, \ldots, \Xi$

$$
|i\rangle=\left|\vec{k}_{i}, \xi_{i}\right\rangle \text {. }
$$

The many-body state $|\Psi\rangle$ is thus an antisymmetrized product of all single-particle states $|i\rangle$ up to the Fermi momenta $\kappa_{\xi}$ of the different components $\xi$. The calculation of the expectation value (5) assuming a cubic box of volume $V$ with periodic boundary conditions is straightforward. Within the Thomas-Fermi approximation $\mathcal{E}_{\text {hom }}$ can be directly translated into the energy density of the inhomogeneous trapped system by replacing the Fermi momenta $\kappa_{\xi}$ with local Fermi momenta $\kappa_{\xi}(\vec{x})$ and adding the contribution of the trapping potential $U_{\xi}(\vec{x})$. This leads to the general form of the energy density of a trapped multicomponent Fermi gas including s- and p-wave interactions [11, 15]

$$
\begin{aligned}
\mathcal{E}\left[\kappa_{1}, \ldots, \kappa_{\Xi}\right](\vec{x}) & =\frac{1}{6 \pi^{2}} \sum_{\xi} U_{\xi}(\vec{x}) \kappa_{\xi}^{3}(\vec{x})+\frac{1}{20 \pi^{2} m} \sum_{\xi} \kappa_{\xi}^{5}(\vec{x})+\frac{a_{0}}{9 \pi^{3} m} \sum_{\xi, \xi^{\prime}>\xi} \kappa_{\xi}^{3}(\vec{x}) \kappa_{\xi^{\prime}}^{3}(\vec{x}) \\
+ & \frac{a_{1}^{3}}{30 \pi^{3} m} \sum_{\xi} \kappa_{\xi}^{8}(\vec{x})+\frac{a_{1}^{3}+b_{0}}{60 \pi^{3} m} \sum_{\xi, \xi^{\prime}>\xi}\left[\kappa_{\xi}^{3}(\vec{x}) \kappa_{\xi^{\prime}}^{5}(\vec{x})+\kappa_{\xi}^{5}(\vec{x}) \kappa_{\xi^{\prime}}^{3}(\vec{x})\right]
\end{aligned}
$$

where the summations range over all components $\xi, \xi^{\prime}=1, \ldots, \Xi$. The interaction is characterized by the s-wave scattering length $a_{0}$, the s-wave effective volume $b_{0}$, and the p-wave scattering length $a_{1}$. In order to keep the treatment as transparent as possible we have assumed equal masses $m$ and interaction parameters for the atoms of all components. The local Fermi momentum $\kappa_{\xi}(\vec{x})$ is connected to the partial density $\rho_{\xi}(\vec{x})$ of the respective component by

$$
\rho_{\xi}(\vec{x})=\frac{1}{6 \pi^{2}} \kappa_{\xi}^{3}(\vec{x}) .
$$

For most of the following investigations the detailed form of the trapping potential $U_{\xi}(\vec{x})$ does not enter. If, however, the shape of the trap is relevant, then we assume a deformed harmonic-oscillator potential

$$
U(\vec{x})=\frac{m}{2}\left(\omega_{1}^{2} x_{1}^{2}+\omega_{2}^{2} x_{2}^{2}+\omega_{3}^{2} x_{3}^{2}\right), \quad \vec{x}=\left(x_{1}, x_{2}, x_{3}\right),
$$

with a mean oscillator frequency $\omega=\sqrt[3]{\omega_{1} \omega_{2} \omega_{3}}$ and the corresponding mean oscillator length $\ell=(m \omega)^{-1 / 2}$. 


\subsection{Functional variation and extremum condition}

On the basis of the energy density functional (7) we obtain the density distribution of the degenerate Fermi gas by functional minimization of the energy

$$
E\left[\kappa_{1}, \ldots, \kappa_{\Xi}\right]=\int \mathrm{d}^{3} x \mathcal{E}\left[\kappa_{1}, \ldots, \kappa_{\Xi}\right](\vec{x}) .
$$

with the constraints of given numbers of atoms $N_{\xi}$ of the different components $\xi=1, \ldots, \Xi$

$$
N_{\xi}=\int \mathrm{d}^{3} x \rho_{\xi}(\vec{x})=\frac{1}{6 \pi^{2}} \int \mathrm{d}^{3} x \kappa_{\xi}^{3}(\vec{x}) .
$$

For the variational treatment these constraints are implemented via a set of Lagrange multipliers $\mu_{\xi}$ - the chemical potentials of the different components. This leads to the Legendre transformed energy

$$
F\left[\kappa_{1}, \ldots, \kappa_{\Xi}\right]=\int \mathrm{d}^{3} x \mathcal{F}\left[\kappa_{1}, \ldots, \kappa_{\Xi}\right](\vec{x}),
$$

where the associated energy density $\mathcal{F}\left[\kappa_{1}, \ldots, \kappa_{\Xi}\right](\vec{x})$ is given by

$$
\mathcal{F}\left[\kappa_{1}, \ldots, \kappa_{\Xi}\right](\vec{x})=\mathcal{E}\left[\kappa_{1}, \ldots, \kappa_{\Xi}\right](\vec{x})-\sum_{\xi} \frac{\mu_{\xi}}{6 \pi^{2}} \kappa_{\xi}^{3}(\vec{x}) .
$$

The set of density distributions $\rho_{\xi}(\vec{x})$ or local Fermi momenta $\kappa_{\xi}(\vec{x})$ that minimizes the transformed energy describes the state of the trapped degenerate Fermi gas. A necessary condition for a minimum of the energy $F\left[\kappa_{1}, \ldots, \kappa_{\Xi}\right]$ is that the derivative of the energy density $\mathcal{F}\left[\kappa_{1}, \ldots, \kappa_{\Xi}\right](\vec{x})$ with respect to the local Fermi momentum vanishes for this set

$$
\frac{\partial}{\partial \kappa_{\xi}(\vec{x})} \mathcal{F}\left[\kappa_{1}, \ldots, \kappa_{\Xi}\right](\vec{x})=0 \quad \text { for all } \quad \vec{x}, \xi .
$$

This condition is also fulfilled by maxima or saddle points, therefore one has to check explicitly whether the solutions describe a minimum of $F\left[\kappa_{1}, \ldots, \kappa_{\Xi}\right]$. Inserting the energy density (13) leads to a set of $\Xi$ coupled polynomial equations for the local Fermi momenta at the point $\vec{x}$

$$
\begin{aligned}
m\left[\mu_{\xi}-U_{\xi}(\vec{x})\right] & =\frac{1}{2} \kappa_{\xi}^{2}(\vec{x})+\frac{2 a_{0}}{3 \pi} \sum_{\xi^{\prime} \neq \xi} \kappa_{\xi^{\prime}}^{3}(\vec{x})+\frac{8 a_{1}^{3}}{15 \pi} \kappa_{\xi}^{5}(\vec{x}) \\
& +\frac{a_{1}^{3}+b_{0}}{30 \pi} \sum_{\xi^{\prime} \neq \xi}\left[3 \kappa_{\xi^{\prime}}^{5}(\vec{x})+5 \kappa_{\xi}^{2}(\vec{x}) \kappa_{\xi^{\prime}}^{3}(\vec{x})\right], \quad \xi=1, \ldots, \Xi .
\end{aligned}
$$

These extremum conditions are the starting point for the investigations on the stability of the trapped Fermi gas presented in the following sections.

\section{Mean-field collapse}

In the presence of attractive interactions between the trapped atoms, i.e., if the (sor p-wave) scattering length is negative, the dilute gas may undergo a collective collapse towards a high-density configuration. The physical origin of the collapse is the attractive mean-field generated by the interaction. At sufficiently low densities this mean-field attraction is compensated by the positive kinetic energy contribution. However, if the density grows the negative mean-field contribution to the energy grows 
faster than the kinetic energy. At some critical density the kinetic part is not able to stabilize the gas against the mean-field attraction and the system collapses. For bosonic systems this phenomenon was observed in a series of outstanding experiments [16].

The same phenomenon can occur in degenerate Fermi gases with attractive s- or p-wave interactions. In the following we derive explicit conditions for the stability of one- and two-component systems.

\subsection{Stability of the single-component Fermi gas}

For the single-component system the Legendre transformed energy density (13) of the multicomponent Fermi gas reduces to

$$
\mathcal{F}_{1}[\kappa](\vec{x})=\frac{1}{6 \pi^{2}}[U(\vec{x})-\mu] \kappa^{3}(\vec{x})+\frac{1}{20 \pi^{2} m} \kappa^{5}(\vec{x})+\frac{a_{1}^{3}}{30 \pi^{3} m} \kappa^{8}(\vec{x}) .
$$

As a consequence of the Pauli principle the s-wave contact interaction does not contribute in a system of identical fermions. However, the p-wave contact interaction does contribute and constitutes the leading interaction term in the single-component gas.

To illustrate the origin of the mean-field instability figure 1 shows the energy density (16) in the centre of the trap as function of the Fermi momentum $\kappa$ for a fixed value of the chemical potential $\mu$ and different p-wave scattering lengths. We express all quantities in units of the atom mass $m$ and a length scale $\ell$, which later on will be the mean oscillator length of the trapping potential. The thin solid curve shows the energy density of a noninteracting Fermi gas $\left(a_{1} / \ell=0\right)$. The minimum defines the actual Fermi momentum at the given position $\vec{x}$ according to the variational description discussed above. If we switch on an attractive p-wave interaction $\left(a_{1} / \ell<0\right)$, then the dominant $\kappa^{8}$ term in (16) leads to a rapid decline of the energy at large Fermi momenta. For sufficiently weak attractions (thick solid curve) a local minimum at low Fermi momenta remains. However, if the strength of the p-wave interaction reaches a critical value the minimum evolves to a saddle point (dashed curve). Beyond this value of the scattering length the energy density drops monotonically and there is no low-density solution any more (dotted curve in figure 1). We conclude that for a given chemical potential there is a limit to the scattering length up to which a metastable low-density solution exists.

The analytic criterion for the instability of the Fermi gas is the vanishing of the local minimum at low densities; the onset of instability is marked by appearance of a the saddle point, where first and second derivative of the transformed energy density $\mathcal{F}_{1}(\kappa)$ vanish. It is advantageous to perform the derivatives of (16) with respect to density $\rho=\kappa^{3} /\left(6 \pi^{2}\right)$ rather than Fermi momentum. This leads to a condition for the existence of the minimum involving the p-wave scattering length $a_{1}<0$ and the local Fermi momentum $\wedge$ 夥

$$
-a_{1} \kappa(\vec{x}) \leq \frac{\sqrt[3]{3 \pi}}{2} \quad \text { and } \quad m[\mu-U(\vec{x})] a_{1}^{2} \leq \frac{3(3 \pi)^{2 / 3}}{40} .
$$

The second inequality is obtained by inserting the Fermi momentum of the inflection point into the extremum condition (15) to obtain a limit for the chemical potential. These are stability conditions for the trapped single-component Fermi gas, in the

$\ddagger$ Alternatively these conditions can be obtained from the extremum condition (15). The collapse region is characterized by parameters where (15) has no real positive solution anymore [11, 15. 


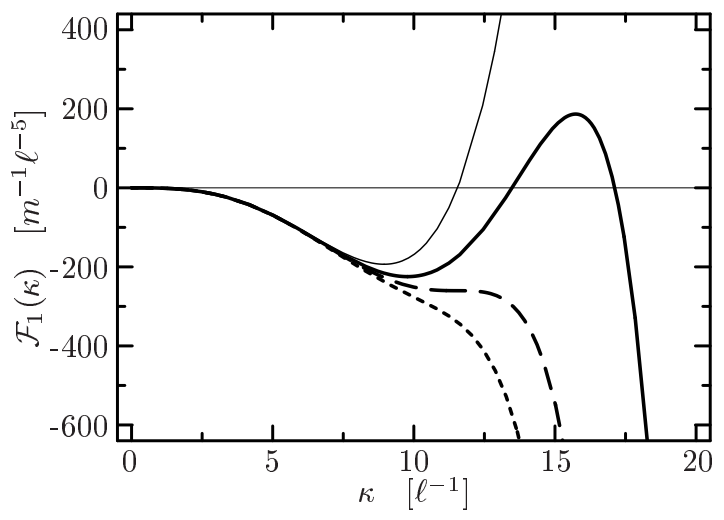

Figure 1. Legendre transformed energy density $\mathcal{F}_{1}(\kappa)$ of the single-component Fermi gas as function of the Fermi momentum $\kappa$ for $m \ell^{2}[\mu-U(\vec{x})]=40$. The different curves correspond to different p-wave scattering lengths: $a_{1} / \ell=0$ (thin solid line), -0.08 (solid), -0.0915 (dashed), and -0.1 (dotted).

presence of an attractive p-wave interaction $a_{1}<0$; any configuration that violates these condition will collapse.

We obtain a more intuitive relation by replacing the Fermi momentum with the total number of atoms $N$ in a trap of given geometry; here we assume a deformed harmonic-oscillator trap (9) with mean oscillator length $\ell$. For a given set of p-wave scattering lengths and the corresponding critical chemical potentials according to (17) we solve the extremum condition numerically and obtain the critical particle number. The resulting relation between $a_{1}$ and the critical particle number $N_{\text {crit }}$ of a metastable trapped Fermi gas can be parametrized by the stability condition

$$
-2.246\left(\sqrt[6]{N} \frac{a_{1}}{\ell}\right) \leq 1
$$

where the prefactor is adjusted according to the pairs of $a_{1}$ and $N_{\text {crit }}$ obtained numerically.

For the single-component Fermi gas the instability induced by attractive p-wave interactions is no severe limitation in the present experimental parameter regime. For typical particle numbers of $N \approx 10^{6}$ and a mean oscillator length of $\ell=1 \mu \mathrm{m}$ the p-wave scattering length would have to be of the order of $a_{1} \approx-840 a_{\mathrm{B}}$ (where $a_{\mathrm{B}}$ is the Bohr radius) to cause the instability. However, by exploiting a p-wave Feshbach resonance [17] these values of the scattering length seem to be reachable.

\subsection{Stability of the two-component Fermi gas}

The same analysis can be performed for a two-component Fermi gas. In order to keep the number of parameters small we assume identical local Fermi momenta $\kappa(\vec{x})=\kappa_{1}(\vec{x})=\kappa_{2}(\vec{x})$ for the two components. A justification of this assumption follows in section 4.1. For these systems the transformed energy density (13) reduces to

$\mathcal{F}_{2}[\kappa](\vec{x})=\frac{1}{3 \pi^{2}}[U(\vec{x})-\mu] \kappa^{3}(\vec{x})+\frac{1}{10 \pi^{2} m} \kappa^{5}(\vec{x})+\frac{a_{0}}{9 \pi^{3} m} \kappa^{6}(\vec{x})+\frac{a_{1}^{3}}{10 \pi^{3} m} \kappa^{8}(\vec{x})$,

where the s-wave effective volume $b_{0}$ is neglected. For this discussion it can be included subsequently through a modified p-wave scattering length $a_{1}^{3} \rightarrow a_{1}^{3}+b_{0} / 3$. In contrast 
to the single-component Fermi gas s-wave as well as p-wave interactions contribute. Their interplay leads to a rich variety of phenomena.

As for the single-component system a mean-field instability of the degenerate gas can emerge as consequence of an attractive interaction. In the two-component case, however, the s-wave or the p-wave interaction may be attractive which causes a more complicated stability condition. Evaluating the Fermi momentum associated with the saddle point of the energy density (19) leads to the stability condition

$$
-a_{0} \kappa(\vec{x})-2\left[a_{1} \kappa(\vec{x})\right]^{3} \leq \frac{\pi}{2} \text {. }
$$

It is convenient to discuss the four possible combinations of signs of the s-wave scattering length $a_{0}$ and the p-wave scattering length $a_{1}$ separately:

$a_{0}>0, a_{1}>0$ : for purely repulsive interactions the gas is stable against collapse for any Fermi momentum.

$a_{0}<0, a_{1}<0$ : for purely attractive interactions both interaction parts cooperate and lower the critical Fermi momentum compared to a pure s-wave or p-wave attraction.

$a_{0}>0, a_{1}<0$ : the repulsive s-wave interaction stabilizes the system against p-wave induced collapse, i.e., the critical Fermi momentum is increased compared to a pure p-wave attraction.

$a_{0}<0, a_{1}>0$ : similar to the previous case the p-wave repulsion stabilizes the system, i.e. increases the critical Fermi momentum, compared to the case of a pure s-wave attraction. Moreover, if the ratio of p-wave and s-wave scattering lengths exceeds a limit given by

$$
\frac{a_{1}}{\left|a_{0}\right|} \geq \frac{2}{3 \pi^{2 / 3}} \approx 0.311,
$$

then the system is absolutely stabilized by the p-wave repulsion. That means, despite of the attractive s-wave interaction the mean-field collapse does not occur. Notice that the p-wave scattering length required for this absolute stabilization is only $1 / 3$ of the s-wave scattering length.

For repulsive p-wave interactions that are slightly to weak to generate absolute stabilization, the density profile of the trapped gas shows a peculiar structure: In a region around the minimum of the trapping potential the density is increased by typically one order of magnitude compared to peripheral regions. In contrast to the outer low-density phase, which is governed by a balance between s-wave attraction and kinetic energy, the inner high-density phase is ruled by the balance between s-wave attraction and p-wave repulsion. Without the p-wave repulsion the high-density phase would collapse immediately. We discuss this phenomenon in detail in ref. 111.

The last class of interactions (attractive s-wave and repulsive p-wave) is of special interest in connection with a BCS phase transition in trapped dilute Fermi gases. The formation of Cooper pairs of two fermionic atoms requires an attractive interaction. One possibility is an attractive s-wave interaction in a two-component Fermi gas which would allow Cooper pairs composed of one atom from each component. The critical temperature for the BCS transition increases with the product $\left|a_{0}\right| \kappa$ of scattering length and Fermi momentum [7]. At the same time the mechanical stability of the system is a prerequisite. This sets an upper limit to the value of $\left|a_{0}\right| \kappa$-and therewith to the BCS transition temperature - described by the stability condition 


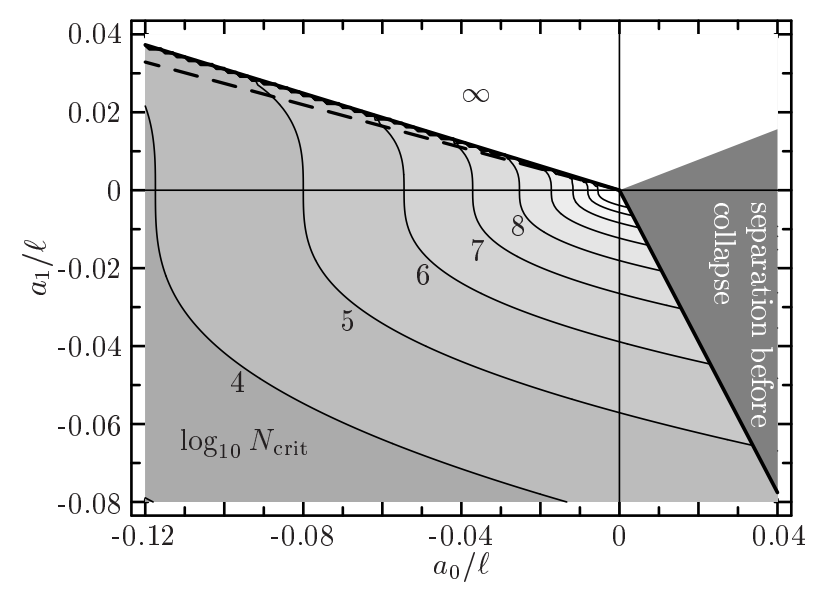

Figure 2. Logarithmic contour plot of the critical particle number $N_{\text {crit }}$ up to which the trapped two-component Fermi gas is stable against mean-field collapse as function of the ratio of s-wave and p-wave scattering lengths to mean oscillator length $\ell$ of the parabolic trap. The white area in the upper part indicates the parameter region, where no collapse occurs.

(20). However, if an appropriate repulsive p-wave interaction is included, then the effect of absolute stabilization can be employed to get rid of this limitation of the transition temperature caused by the mean-field instability $\$$.

An overview of the influence of s- and p-wave interactions on the stability of the two-component Fermi gas against mean-field induced collapse is given in figure 2. The contour plot shows the critical particle number $N_{\text {crit }}$ for a trap of mean oscillator length $\ell$ as function of the ratios $a_{0} / \ell$ and $a_{1} / \ell$. The particle number is calculated numerically in the same way as described for the single-component gas [11, 15. The plot confirms the relevance of the p-wave part of the interaction for the propertiesespecially for the stability - of the dilute degenerate Fermi gas. Two major effects of the p-wave interaction are: (i) Absolute stabilization of the system by repulsive $\mathrm{p}$-wave interactions in the presence of a s-wave attraction (white region) and p-wave stabilized high-density phase (region between the dashed line and the onset of absolute stabilization). (ii) Mean-field collapse induced by p-wave attraction also for systems with repulsive s-wave interactions (lower right quadrant). In addition a competition with another effect, the component separation that is discussed in the following section, appears (dark gray area).

As a rule of thumb, the p-wave part becomes relevant as soon as the p-wave scattering length exceeds about $1 / 10$ of the value of the s-wave scattering length. A detailed discussion of the various effects is presented in [1].

\section{Component separation}

A second structural transition that can occur in degenerate two-component Fermi gases is the spatial demixing of the two components 18, 19. The physical mechanism behind this transition can be understood from the general form of the energy density

$\S$ Of course it has to be checked that the gain of mechanical stability due to the p-wave repulsion is not counteracted by a destructive effect on the Cooper pairs. 


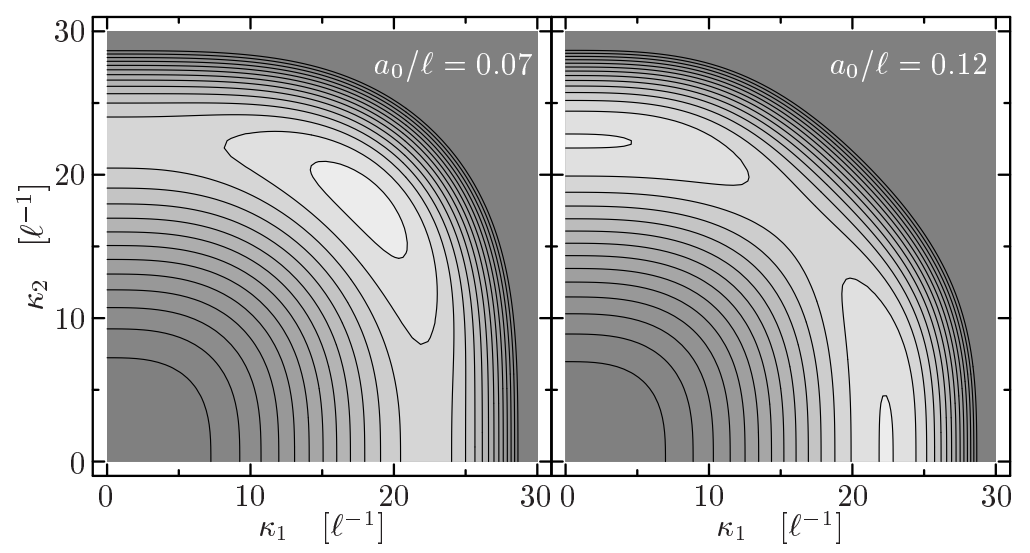

Figure 3. Contour plots of the local energy density $\mathcal{F}_{2}\left(\kappa_{1}, \kappa_{2}\right)$ of a twocomponent Fermi gas in the centre of the trap as function of the Fermi momenta $\kappa_{1}$ and $\kappa_{2}$ for $\mu_{1}=\mu_{2}=250 \mathrm{~m}^{-1} \ell^{-2}$. The left panel shows the case of a moderate s-wave repulsion $\left(a_{0} / \ell=0.07, a_{1} / \ell=0\right)$ that still supports identical density distributions for both components. The right panel corresponds to a stronger s-wave repulsion $\left(a_{0} / \ell=0.12, a_{1} / \ell=0\right)$ which forces the components to demix partially. Brighter shading corresponds to smaller values of the energy density.

of a two-component system resulting from (13)

$$
\begin{aligned}
\mathcal{F}_{2}\left[\kappa_{1}, \kappa_{2}\right](\vec{x})= & \frac{1}{6 \pi^{2}}\left[\left(U_{1}(\vec{x})-\mu_{1}\right) \kappa_{1}^{3}(\vec{x})+\left(U_{2}(\vec{x})-\mu_{2}\right) \kappa_{2}^{3}(\vec{x})\right] \\
& +\frac{1}{20 \pi^{2} m}\left[\kappa_{1}^{5}(\vec{x})+\kappa_{2}^{5}(\vec{x})\right]+\frac{a_{0}}{9 \pi^{3} m} \kappa_{1}^{3}(\vec{x}) \kappa_{2}^{3}(\vec{x}) \\
& +\frac{a_{1}^{3}}{30 \pi^{3} m}\left[\kappa_{1}^{8}(\vec{x})+\kappa_{2}^{8}(\vec{x})+\frac{1}{2} \kappa_{1}^{3}(\vec{x}) \kappa_{2}^{5}(\vec{x})+\frac{1}{2} \kappa_{1}^{5}(\vec{x}) \kappa_{2}^{3}(\vec{x})\right] .
\end{aligned}
$$

The driving term for component separation is the s-wave part of the interaction. Its contribution to the energy density is proportional to the product of the densities of both components $\rho_{1}(\vec{x}) \rho_{2}(\vec{x}) \propto \kappa_{1}^{3}(\vec{x}) \kappa_{2}^{3}(\vec{x})$. For strongly repulsive s-wave interactions it is energetically favourable to spatially separate the two components such that the overlap of the two density distributions and thus the contribution of the s-wave part to the total energy is reduced.

A quantitative example is shown in figure 3. The contour plots depict the local energy density $\mathcal{F}_{2}\left(\kappa_{1}, \kappa_{2}\right)$ for a fixed value of $\mu_{1}=\mu_{2}$ as function of the two Fermi momenta $\kappa_{1}$ and $\kappa_{2}$ for two different positive s-wave scattering lengths $a_{0}$. The left panel $\left(a_{0} / \ell=0.07\right)$ depicts a standard case where the energy density has a single minimum at $\kappa_{1}=\kappa_{2} \neq 0$. This class of solutions with identical density profiles for both components was already discussed in the context of collapse due to attractive interactions.

A new class of solutions appears, if the strength of the s-wave repulsion is increased further. As shown in the right panel of figure $3\left(a_{0} / \ell=0.12\right)$ the minimum at equal Fermi momenta has evolved into a saddle point and two energetically degenerate minima emerge at $\kappa_{1}=0, \kappa_{2}>0$ and $\kappa_{1}>0, \kappa_{2}=0$, respectively. For these interaction strengths the density of one component is depleted or even zero and the density of the other one is increased in a central region of the trap. 


\subsection{Stability conditions}

The onset of component demixing is indicated by the transition of the minimum at $\kappa_{1}=\kappa_{2}$ into a saddle point. We can derive a relation that characterizes the onset of component separation by looking at the determinant of the Hesse second derivative matrix

$$
D\left(\rho_{1}, \rho_{2}\right)=\left|\begin{array}{cc}
\frac{\partial^{2} \mathcal{F}_{2}}{\partial \rho_{1}^{2}} & \frac{\partial^{2} \mathcal{F}_{2}}{\partial \rho_{1} \partial \rho_{2}} \\
\frac{\partial^{2} \mathcal{F}_{2}}{\partial \rho_{2} \partial \rho_{1}} & \frac{\partial^{2} \mathcal{F}_{2}}{\partial \rho_{2}^{2}}
\end{array}\right|,
$$

where in $\mathcal{F}_{2}\left[\kappa_{1}\left(\rho_{1}\right), \kappa_{2}\left(\rho_{2}\right)\right]$ the Fermi momenta are expressed in terms of the densities. Again, it is convenient to use derivatives with respect to the densities, since this eliminates the dependence of the transformed energy on the chemical potential automatically. Those stationary points of the energy density that are minima (or maxima) yield $D\left(\rho_{1}, \rho_{2}\right)>0$ whereas saddle points are characterized by $D\left(\rho_{1}, \rho_{2}\right)<0$. The parameter sets where the transition from a minimum at $\rho=\rho_{1}=\rho_{2}$ to a saddle point happens are determined by $D(\rho, \rho)=0$. This is a straightforward generalization of the procedure used in section 3.2 to obtain the stability condition for mean-field collapse. Inserting the energy density (22) into (23) leads to

$$
\left[2 a_{0} \kappa+4\left(a_{1} \kappa\right)^{3}+\pi\right]\left[-2 a_{0} \kappa+2\left(a_{1} \kappa\right)^{3}+\pi\right]=0 .
$$

The first factor resembles the stability condition (20) for mean-field collapse of the Fermi gas. The second factor gives a stability condition for component demixing. The configuration of overlapping identical density profiles of the two-components is stable only if the condition

$$
a_{0} \kappa(\vec{x})-\left[a_{1} \kappa(\vec{x})\right]^{3} \leq \frac{\pi}{2}
$$

is fulfilled everywhere in the trap. In regions of the trap where this condition is violated the overlapping configuration is unstable against demixing of the components.

As in the case of collapse it is convenient to consider the different combinations of signs of the s- and p-wave scattering lengths separately:

$a_{0}>0, a_{1}>0$ : with increasing s-wave scattering length the critical density for component separation decreases. A repulsive p-wave interaction stabilizes, i.e., it increases the critical density. If the ratio of p-wave and s-wave scattering length exceeds the limit

$$
\frac{a_{1}}{a_{0}} \geq \frac{2^{4 / 3}}{3 \pi^{2 / 3}} \approx 0.392,
$$

then the overlapping configuration is absolutely stabilized; no component separation occurs at all.

$a_{0}>0, a_{1}<0$ : the attractive p-wave interaction destabilizes, i.e., reduces the maximum density of the overlapping configuration. If the p-wave attraction is sufficiently strong such that

$$
\frac{\left|a_{1}\right|}{a_{0}}>2(3 / \pi)^{2 / 3} \approx 1.939,
$$

then the critical density for a mean-field collapse is lower than the critical density for component separation. Therefore no component separation but a mean-field collapse of the overlapping configuration occurs.

$a_{0} \leq 0$ : component separation does not occur, even if the p-wave interaction is strongly repulsive. 


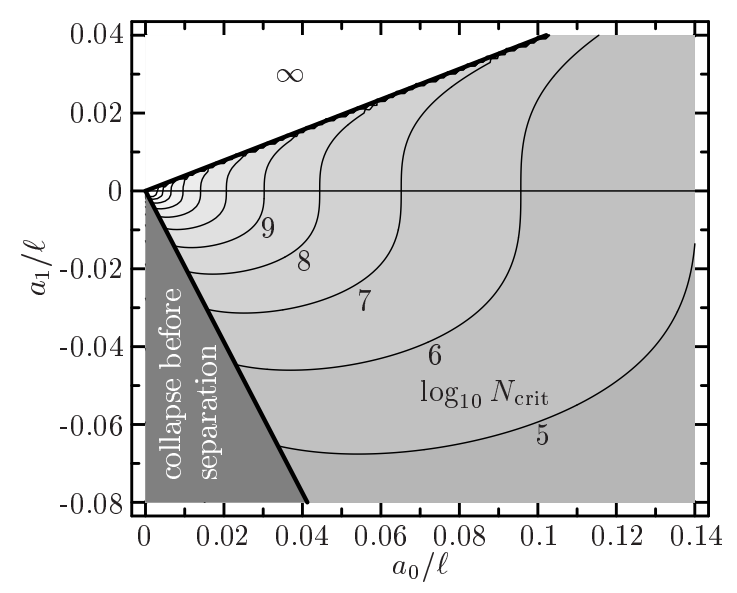

Figure 4. Logarithmic contour plot of the critical particle number $N_{\text {crit }}$ up to which the trapped two-component Fermi gas is stable against component separation as function of the ratio of s-wave and p-wave scattering lengths to mean oscillator length $\ell$ of the parabolic trap. The white area in the upper part indicates the parameter region, where no separation occurs.

The twisted behaviour of the p-wave interaction with respect to component separation can be understood from the structure of the energy density (22). The driving force of component separation - the s-wave interaction - contributes a term proportional to the product of the densities of both components. Thus for repulsive s-wave interactions a configuration with fully overlapping density distributions is higher in energy than a separated configuration with small overlap but higher absolute densities. The pwave interaction, however, has a term $\left[\rho_{1} \rho_{2}^{5 / 3}+\rho_{1}^{5 / 3} \rho_{2}\right]$ proportional to the product of the densities to some power and an additional term that depends on the sum of the densities, $\left[\rho_{1}^{8 / 3}+\rho_{2}^{8 / 3}\right]$. Thus a strongly repulsive p-wave interaction disfavours both, large overlaps of the two density distributions and large densities of a single component. Therefore a p-wave repulsion alone cannot induce component separation. Moreover, it stabilizes against separation in connection with a repulsive s-wave interaction.

A pictorial overview of the influence of s- and p-wave interactions on the onset of component separation is given by the stability map in figure 4 . The contour plot shows the logarithm of the critical particle number $N_{\text {crit }}$ as function of the s- and pwave scattering lengths. This critical particle number is determined from the solution of the extremum condition (15) that reaches the critical Fermi momentum (25) in the trap centre. Like in section 3.2 a deformed parabolic trap of mean oscillator length $\ell$ is assumed. As soon as the particle number $N=N_{1}=N_{2}$ exceeds the critical particle number $N_{\text {crit }}$ a demixing of the components appears in the trap centre. The plot clearly reveals the influence of the p-wave interaction: Attractive p-wave interactions, on the one hand, reduce the critical particle number. Moreover, for those interaction parameters in the dark gray area the p-wave attraction is so strong that the mean-field collapse of the overlapping configuration happens before the critical density for component separation is reached. Repulsive p-wave interactions, on the other hand, stabilize the overlapping configuration against component separation and prevent separation completely for interaction parameters in the white region in figure A. 


\subsection{Structure of the separated configurations}

With a few examples we want to discuss typical shapes of the demixed density distributions that appear beyond the critical particle number. To discuss the general phenomenology we restrict ourselves to pure s-wave interactions $\left(a_{1} / \ell=0\right)$ and equal particle numbers $N=N_{1}=N_{2}$ in a spherical symmetric trapping potential.

Even for fixed particle numbers and interaction strengths there exists a rich variety of different density distributions that may appear in case of component separation. In general it depends on the detailed dynamics of the system which of the energetically (almost) degenerate states is realized. We can distinguish two major classes of separated solutions: Those with equal chemical potentials $\mu_{1}=\mu_{2}$ for both components and those with $\mu_{1} \neq \mu_{2}$.

For equal chemical potentials $\mu_{1}=\mu_{2}$ [more precisely $\left.\mu_{1}-U_{1}(\vec{x})=\mu_{2}-U_{2}(\vec{x})\right]$ the extremum condition (15) is symmetric with respect to exchange of the two components. If the s-wave scattering length is large enough for phase demixing then there are two energetically degenerate solutions $\left\{\rho_{1}^{(A)}(\vec{x}), \rho_{2}^{(A)}(\vec{x})\right\}$ and $\left\{\rho_{1}^{(B)}(\vec{x}), \rho_{2}^{(B)}(\vec{x})\right\}$ (compare figure 3), where

$$
\rho_{1}^{(\mathrm{A})}(\vec{x})=\rho_{2}^{(\mathrm{B})}(\vec{x}), \quad \rho_{2}^{(\mathrm{A})}(\vec{x})=\rho_{1}^{(\mathrm{B})}(\vec{x}) .
$$

In general to construct density profiles with equal number of particles in both components we can switch between the two solutions depending on the position in the trap. The most simple ansatz is to take solution (A) in one hemisphere and (B) in the other

$$
\rho_{\xi}^{\text {axial }}(r, z)= \begin{cases}\rho_{\xi}^{(\mathrm{A})}\left(\sqrt{r^{2}+z^{2}}\right) & \text { for } z \geq 0 \\ \rho_{\xi}^{(\mathrm{B})}\left(\sqrt{r^{2}+z^{2}}\right) & \text { for } z<0\end{cases}
$$

which guarantees $N_{1}=N_{2}$. Examples for the density profiles $\rho_{1}^{\text {axial }}(r, z)$ along the $z$ axis obtained for different s-wave scattering lengths are shown in figure 5 . The upper left panel corresponds to an interaction strength that is slightly to weak to cause separation. A tiny increase of $a_{0} / \ell$ leads to the onset of component separation (upper right panel), a small density wiggle appears on top of the smooth density profile in the trap centre. Further increase of the s-wave repulsion enhances this demixing and finally leads to a nearly complete spatial separation where one component is localized in one hemisphere and the second one in the other hemisphere (lower right panel).

The ansatz (29) is by no means unique. One could subdivide the trap volume into several domains and switch between the two solutions in each of them. For example, in a spherical trap one could imagine domains that look like pieces of a cutted pie. Within the Thomas-Fermi approximation the total energy is not affected. However, if we go beyond the Thomas-Fermi approximation, e.g. by including gradient corrections to the kinetic energy, the strong density variations at the interface of the domains produce a positive energy contribution. For typical particle numbers of $10^{6}$ the relative contribution of the gradient corrections for a cutted distribution like (29) is in the order of $10^{-5}$ [14]. Even though this is negligible, we assume that the configuration with minimal interface is preferred - the axially symmetric profile (29) in this case.

There is also a class of spherically symmetric solutions with $N_{1}=N_{2}$ that was discussed by several authors [18, 19]. However, it turns out that these spherical solutions are higher in energy than the axial ones. In figure 6 two examples for these spherical configurations are shown. The plots also contain the respective values 


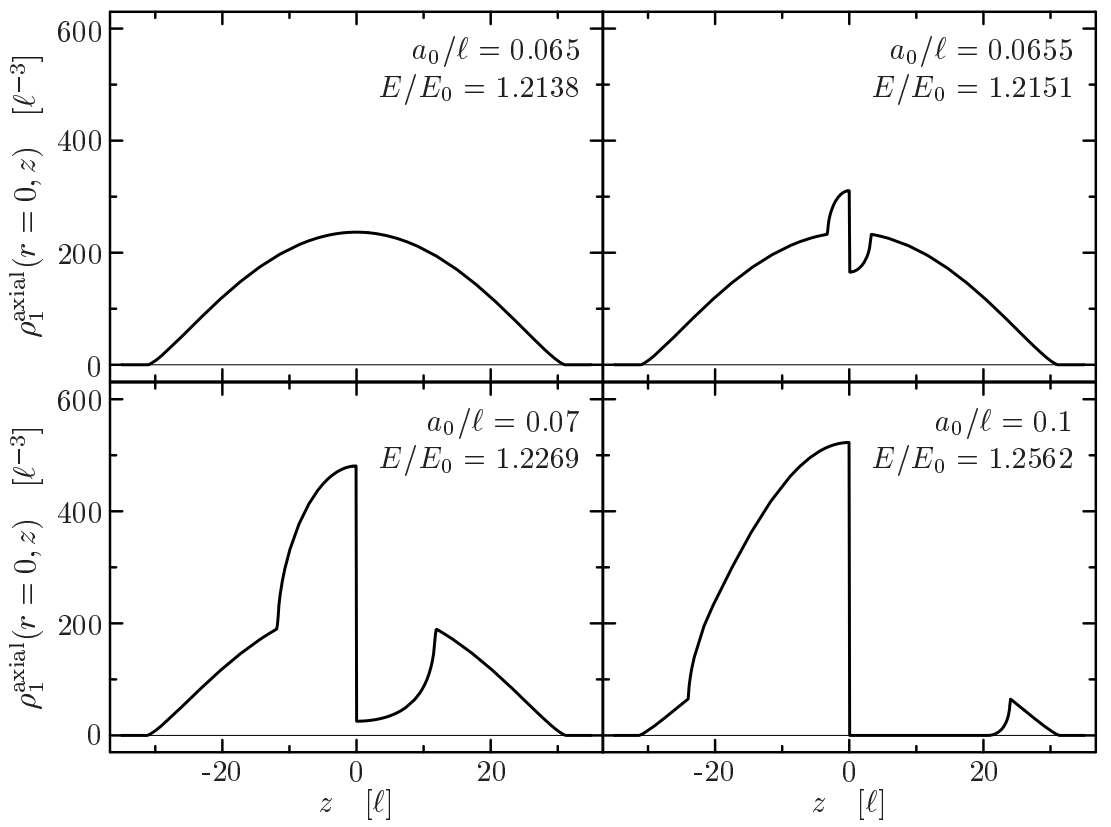

Figure 5. Axially symmetric configuration: density profile $\rho_{1}^{\text {axial }}(r=0, z)$ along the $z$-axis for two-component Fermi gases with $N_{1}=N_{2}=10^{7}$ and different s-wave scattering lengths $a_{0} / \ell$. The distribution of the second component $\rho_{2}^{\text {axial }}(r=0, z)=\rho_{1}^{\text {axial }}(r=0,-z)$ is obtained by inversion of the ordinate. An animated GIF of the density distributions is available from the article's abstract page in the online journal [2].

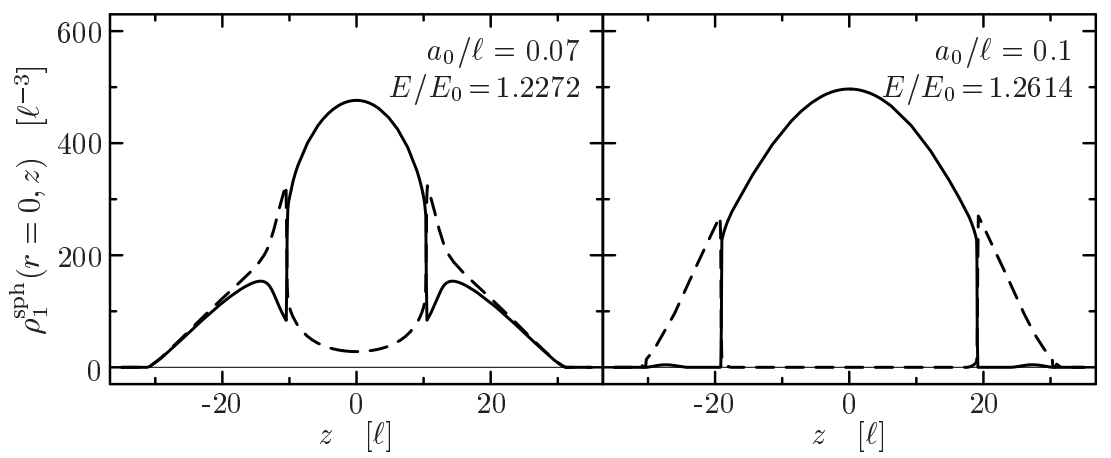

Figure 6. Spherical configuration: density profiles $\rho_{1}^{\mathrm{sph}}(r=0, z)$ (solid line) and $\rho_{2}^{\mathrm{sph}}(r=0, z)$ (dashed line) along the $z$-axis with $N_{1}=N_{2}=10^{7}$ for different s-wave scattering lengths $a_{0} / \ell$.

of the total energy in units of the energy $E_{0}=\frac{3 N}{2 m \ell^{2}} \sqrt[3]{6 N}$ of the noninteracting twocomponent gas with the same particle number. For comparison the energies of the axially symmetric configurations are given in figure 5. It should be noted that in the spherical case the components have slightly different chemical potentials.

In an equilibrium situation or during a quasi-stationary process that leads from overlapping density distributions $\rho_{1}(\vec{x})=\rho_{2}(\vec{x})$ to a separated configuration, e.g. by slowly increasing the s-wave scattering length using a Feshbach resonance [17], we expect the energetically lowest, i.e. the axial configuration with least interface shown 


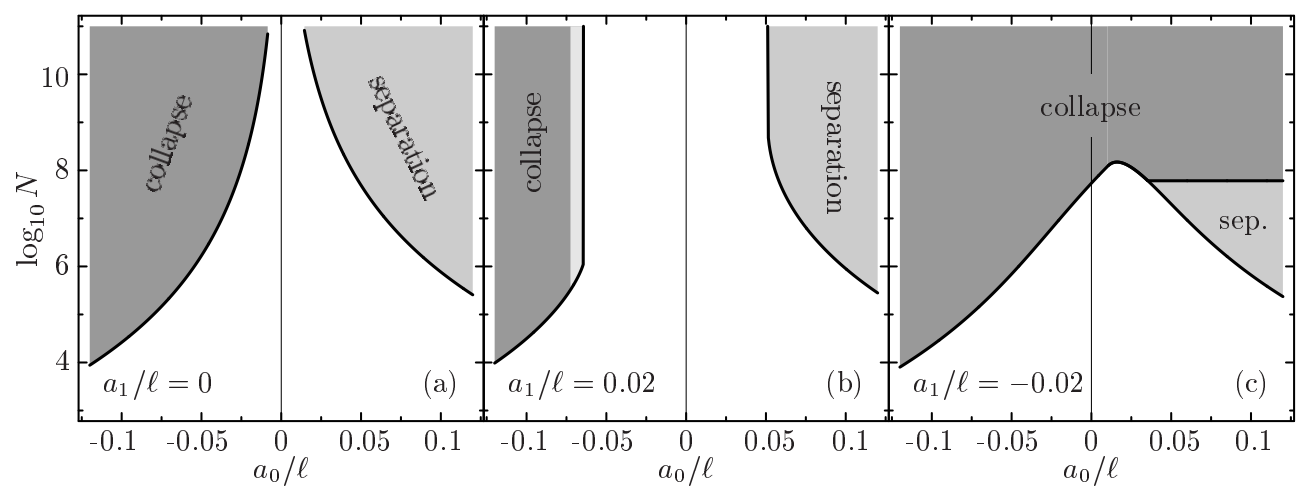

Figure 7. Zero-temperature phase diagrams of a two-component Fermi gas in the parameter plane spanned by s-wave scattering length $a_{0} / \ell$ and particle number $N=N_{1}=N_{2}$. The three diagrams correspond to different values of the pwave scattering length: (a) $a_{1} / \ell=0$, (b) $a_{1} / \ell=0.02$, (c) $a_{1} / \ell=-0.02$. The white area marks the parameter region where both components show identical overlapping density profiles. The dark gray area indicates the region of mean-field collapse and the light gray region characterizes parameter combinations where both components separate (see text). An animated GIF of the phase diagram is available from the article's abstract page in the online journal [20].

in figure 5 , to be realized. This evolution of the density distributions is illustrated by an animation that is available from the article's abstract page in the online journal or from 20].

\section{Summary: Phase diagram of trapped degenerate Fermi gases}

To summarize our findings on the stability against mean-field collapse and component separation we construct a phase diagram of the two-component Fermi gas at zero temperature. The relevant parameters are the s- and p-wave scattering lengths and the particle number $N=N_{1}=N_{2}$. Figure 0 shows the phase diagram in the plane spanned by the s-wave scattering length $a_{0} / \ell$ and the logarithm of the particle number $N$ for three different values of the p-wave scattering length $a_{1} / \ell$. We can identify three classes of density distributions - or phases - of the trapped twocomponent Fermi gas: identical overlapping density distributions for both components (white region), partially or completely demixed density profiles (gray region), and the p-wave stabilized high-density phase (light gray area). In addition the collapse of the system due to an attractive mean-field can occur (dark gray).

In absence of the p-wave interaction the phase diagram has a rather simple structure shown in part (a) of figure \&. If the s-wave interaction is repulsive $\left(a_{0}>0\right)$ the system enters the separated phase if the quantity $\sqrt[6]{N} a_{0} / \ell$ exceeds a limiting value. Similarly the gas collapses in the presence of attractive s-wave interactions if the quantity $\sqrt[6]{N} a_{0} / \ell$ exceeds a another maximum value.

The inclusion of p-wave interactions modifies this picture significantly. Panel (b) of figure 7 depicts the phase diagram for a rather weak repulsive p-wave interaction with $a_{1} / \ell=0.02$. For s-wave interaction strengths below a certain limit the overlapping phase extents up to very high particle numbers; the p-wave repulsion generates absolute stabilization against collapse and component separation. Moreover, the p-wave stabilized high density phase emerges near the threshold for absolute 
stabilization against collapse (light gray region).

Dramatic effects on the phase diagram are caused also by weak attractive pwave interactions as shown in part (c). The maximum particle numbers possible in the overlapping phase are significantly reduced, i.e. the p-wave attraction promotes collapse as well as component separation. In addition it causes the collapse of the separated configuration, an effect that is in close relation to the collapse of a single component Fermi gas. If the density or Fermi momentum of one of the components in the separated configuration exceeds the critical value given by $(17)$ then this component will collapse. Actually this causes a distinct reduction of the parameter region where a stable separated configuration occurs.

Our investigations show that the p-wave interaction can have big influence on the properties of trapped degenerate Fermi gases. In the single-component system it is the origin of the mean-field instability. In a two-component systems it modifies the phase diagram substantially and gives rise to completely new phenomena like the absolute stabilization against s-wave induced collapse and separation. In this way the p-wave interaction may be very useful for the envisioned transition to a superfluid state in trapped dilute Fermi gases.

\section{References}

[1] Inguscio M, Stringari S and Wieman C E (Eds.) 1999 Proceedings of the International School of Physics "Enrico Fermi" (Amsterdam: IOS Press)

[2] DeMarco B and Jin D S 1999 Science 2851703

[3] Truscott A G, Strecker K E, McAlexander W I et al 2001 Science 2912570

[4] Schreck F, Khaykovich L, Corwin K L et al 2001 Phys. Rev. Lett. 87080403

[5] Donley E A, Claussen N R, Cornish S L, et al 2001 Nature 412295

[6] Onofrio R, Durfee D S , Raman C et al 2000 Phys. Rev. Lett. 84810

[7] Houbiers M, Ferwerda R, Stoof H T C et al 1997 Phys. Rev. A 564864

[8] Stoof H T C and Houbiers M 1999 in [1] 537

[9] Roth R and Feldmeier H 2001 submitted to Phys. Rev. B; Preprint cond-mat/0107007

[10] Feldmeier H, Neff T, Roth R and Schnack J 1998 Nucl. Phys. A632 61

[11] Roth R and Feldmeier H 2001 to be published in Phys. Rev. A 64; Preprint cond-mat/0102416

[12] Huang K and Yang C N 1957 Phys. Rev. 105767

[13] Dalfovo F, Giorgini S, Pitaevskii L P and Stringari S 1999 Rev. Mod. Phys. 71463

[14] Roth R 2000 PhD thesis, Tech. Universität Darmstadt, URL http://theory.gsi.de/ rroth/phd

[15] Roth R and Feldmeier H 2000 J. Phys. B 33 L787

[16] Roberts J L, Claussen N R, Cornish S L et al 2001 Phys. Rev. Lett. 864211

[17] Bohn J L 2000 Phys. Rev. A 61053409

[18] Salasnich L, Pozzi B, Parola A and Reatto L 2000 J. Phys. B 333943

[19] Amoruso A, Meccoli I, Minguzzi A and Tosi M P 2000 Eur. Phys. J. D 8361

[20] Several related multimedia supplements are available at the URL http://theory.gsi.de/ ${ }^{\sim}$ trap 